\title{
A Property on Singularities of NURBS Curves
}

\author{
A. Arnal ${ }^{1}$, A. Lluch ${ }^{1}$, and J. Monterde ${ }^{2}$ \\ 1 Dep. de Matemàtiques,Universitat Jaume I \\ Castelló, Spain parnal@mat.uji.es,lluch@mat.uji.es \\ 2 Dep. de Geometria i Topologia, Universitat de València, \\ Burjassot (València), Spain monterde@uv.es
}

\begin{abstract}
We prove that if an open Non Uniform Rational B-Spline curve of order $k$ has a singular point, then it belongs to both curves of order $k-1$ defined in the $k-2$ step of the de Boor algorithm. Moreover, both curves are tangent at the singular point.
\end{abstract}

\section{Introduction}

There are some references in the literature (see [1], [7] or [2]) dealing with methods and algorithms to detect singularities on Bézier curves and its generalizations. Another approach to the study of singularities in polynomial curves is to deduce some properties of the curve when a singularity is present. This is what is done in [5] where an analysis of the behavior of rational Bézier curves under the change of one of its control points was realized. One of the properties of singular Rational Bézier curves shown in [5] is that given a $n$th degree singular Rational Bézier curve the singular point belongs to the $(n-1)$ th degree rational Bézier curve associated with the first $n$ control points and the corresponding weights and also to the $(n-1)$ th degree rational Bézier curve associated to the last $n$ control points and corresponding weights. Moreover, both curves, which are both $(n-1)$ th degree rational Bézier curves defined in the $(n-1)$ th step of the de Casteljau algorithm, intersect tangentially.

This note presents a generalization of some of the properties on [5] for the casc of B-Spline curves and Nurbs curves. In particular we obtain that if an open Nurbs curve of order $k$ has a singular point, then it belongs, analogously to the rational Bézier case, to both curves of order $k-1$ defined in the $k-2$ step of the de Boor algorithm. Morcover, we found that both curves are also tangent at the singular point.

\section{Definitions and properties of Nurbs curves}

\subsection{Definition of Nurbs and B-Spline curves}

According to [4] a B-Spline of order $k$ is made up of pieces of polynomials of degree $k-1$, joined together with $C^{k-2}$ continuity at the break points, so, 
corresponding to the set of break points $t_{0}<t_{1}<t_{2}<\ldots<t_{m-1}<t_{m}$ of a $B-S p l i n e$ function, it's defined the associated knot vector

$$
T=\left(t_{0}, t_{1}, t_{2}, \ldots, t_{m-1}, t_{m}\right)
$$

We refer to the individual points $t_{k}$ of $T$ as knots.

Thus, given a knot vector $T=\left(t_{0}, t_{1}, \ldots, t_{n-1}, t_{n}, t_{n+1}, \ldots, t_{n+k}\right)$ the associated normalized B-Spline, $N_{i, k}$, of order $k$ (degree $k-1$ ) is defined to be the following function:

$$
N_{i, 1}(t)=\left\{\begin{array}{lr}
1 & \text { for } t_{i} \leq t<t_{i+1} \\
0 & \text { otherwise }
\end{array}\right.
$$

for $k=1$, and

$$
N_{i, k}(t)=\frac{\left(t-t_{i}\right)}{\left(t_{i+k-1}-t_{i}\right)} N_{i, k-1}(t)+\frac{\left(t_{i+k}-t\right)}{\left(t_{i+k}-t_{i+1}\right)} N_{i+1, k-1}(t),
$$

$\forall k>1$ and $\forall i=1, \ldots, n$.

The main properties of the basis functions we will use in this note are:

1. $N_{i, k}(t)>0$ for $t \in\left[t_{i}, t_{i+k}\right]$ and $N_{i, k}(t)=0$ otherwise.

2. $\sum_{i=0}^{n} N_{i, k}(t)=1 \quad \forall t \in\left[t_{k-1}, t_{n+1}\right]$.

Now, let us introducc the definition of a Nurbs curve. According to [6], a Non Uniform Rational B-Spline curve of order $k$ (Nurbs), is defined by

$$
X(t)=\frac{\sum_{i=0}^{n} N_{i, k}(t) w_{i} D_{i}}{\sum_{i=0}^{n} N_{i, k}(t) w_{i}} \quad \forall t \in\left[t_{k-1}, t_{n+1}\right] \text { and } n \geqslant k-1
$$

where the $\left\{D_{i}\right\}_{i=0}^{n}$ are the control points, the $\left\{w_{i}\right\}_{i=0}^{n}$ are the weights and the $N_{i, k}$ are the normalized B-Spline functions of order $k$ defined on the knot vector

$$
T=\left(t_{0}, t_{1}, \ldots, t_{n-1}, t_{n}, t_{n+1}, \ldots, t_{n+k}\right) .
$$

Along this note we have chosen the knot vector

$$
T=\left(t_{0}=t_{1}=\ldots=t_{k-1}<t_{k}<\ldots<t_{n}<t_{n+1}=t_{n+2}=\ldots=t_{n+k}\right)
$$

to get an open and clamped Nurbs curve of order $k$ with endpoints $D_{0}$ and $D_{n}$. Remark 1. If we choose weights $w_{i}=1 \forall i$, then, from the second property of the basis functions we get that Nurbs curves are a generalization of B-Spline curves.

Therefore a B-Spline curve is defined as follows:

Given the control points $\left\{D_{i}\right\}_{i=0}^{n}$ a B-Spline curve of order $k, k \leqslant n-1$, associated to the knot vector, $T$, as above, is defined as

$$
X(t)=\sum_{i=0}^{n} N_{i, k}(t) D_{i}, \quad \forall t \in\left[t_{k-1}, t_{n+1}\right] .
$$




\subsection{The de Boor algorithm for Nurbs curves}

For the practical evaluation of Nurbs curves with given weights, we can use the de Boor algorithm, (scc [4]), which allows the computation of points on a Nurbs curve without explicit knowledge of the B-Spline basis functions $\left\{N_{i, k}\right\}$. The algorithm construction is based on the recursive definition of the normalized B-Spline.

Taking the initial values

$$
D_{j}^{0}(t)=D_{j}, \quad w_{j}^{0}(t)=w_{j},
$$

and defining

$$
\alpha_{i}^{j}(t)=\frac{t-t_{i}}{t_{i+k-j}-t_{i}}
$$

the scheme of computation is the following

$$
\begin{array}{rlr}
w_{i}^{j}(t) D_{i}^{j}(t) & =\left(1-\alpha_{i}^{j}(t)\right) w_{i-1}^{j-1}(t) D_{i-1}^{j-1}(t)+\alpha_{i}^{j}(t) w_{i}^{j-1}(t) D_{i}^{j-1}(t) \\
w_{i}^{j}(t) & =\left(1-\alpha_{i}^{j}(t)\right) w_{i-1}^{j-1}(t)+\alpha_{i}^{j}(t) w_{i}^{j-1}(t) & j>0
\end{array}
$$

where, in addition to the weighted de Boor points, we also apply the algorithm to the weights.

Then, after $k-1$ steps, it finally leads to

$$
X(t)=\frac{w_{r}^{k-1}(t) D_{r}^{k-1}(t)}{w_{r}^{k-1}(t)}=D_{r}^{k-1}(t), \quad t \in\left[t_{r} ; t_{r+1}\right]
$$

On the other hand, according to [6], we will express the first derivative of a Nurbs curve

$$
X(t)=\frac{\sum_{i=0}^{n} N_{i, k}(t) w_{i} D_{i}}{\sum_{i=0}^{n} N_{i, k}(t) w_{i}}=\frac{A(t)}{w(t)},
$$

by the following formula

$$
X^{\prime}(t)=\frac{A^{\prime}(t)-w^{\prime}(t) X(t)}{w(t)}
$$

where

$$
\begin{aligned}
& A^{\prime}(t)=(k-1) \sum_{i=1}^{n} \frac{w_{i} D_{i}-w_{i-1} D_{i-1}}{t_{i+k-1}-t_{i}} N_{i, k-1}(t) \\
& w^{\prime}(t)=(k-1) \sum_{i=1}^{n} \frac{w_{i}-w_{i-1}}{t_{i+k-1}-t_{i}} N_{i, k-1}(t)
\end{aligned}
$$




\subsection{The blossoming principle for Nurbs curves}

According to the blossoming principle for B-Spline curves at [4], we can state a generalization for Nurbs curves. This blossoming principle is based on the construction of the polar form associated to the B-Spline basis functions, and it uses its recursive formula (1).

If we define

$$
\begin{aligned}
& w_{i}^{j}\left[u_{0}, u_{1}, \ldots, u_{j-1}\right]:= \\
& \frac{\left(t_{i+k-j}-u_{j-1}\right) w_{i-1}^{j-1}\left[u_{0}, u_{1}, \ldots, u_{j-2}\right]+\left(u_{j-1}-t_{i}\right) w_{i}^{j-1}\left[u_{0}, u_{1}, \ldots, u_{j-2}\right]}{t_{i+k-j}-t_{i}},
\end{aligned}
$$

and $D_{i}^{j}\left[u_{0}, u_{1}, \ldots, u_{j-1}\right]$ such that

$$
\begin{gathered}
w_{i}^{j}\left[u_{0}, u_{1, \ldots,} u_{j-1}\right] D_{i}^{j}\left[u_{0}, u_{1}, \ldots, u_{j-1}\right]= \\
\frac{\left(t_{i+k-j}-u_{j-1}\right) w_{i-1}^{j-1}\left[u_{0}, u_{1, \ldots}, u_{j-1}\right] D_{i-1}^{j-1}\left[u_{0}, u_{1}, \ldots, u_{j-2}\right]}{t_{i+k-j}-t_{i}} \\
+\frac{\left(u_{j-1}-t_{i}\right) w_{i}^{j-1}\left[u_{0}, u_{1}, \ldots, u_{j-1}\right] D_{i}^{j-1}\left[u_{0}, u_{1}, \ldots, u_{j-2}\right]}{t_{i+k-j}-t_{i}}
\end{gathered}
$$

then, the blossoming principle asserts that the Nurbs curve is given by

$$
X(t)=\frac{w_{r}^{k-1}\left[t,{ }^{(k-1)} ., t\right] D_{r}^{k-1}\left[t, .^{(k-1)} ., t\right]}{w_{r}^{k-1}\left[t,{ }^{(k-1)} ., t\right]}=D_{r}^{k-1}\left[t,{ }^{(k-1)} ., t\right] .
$$

\section{Singularities of Nurbs curves}

A differentiable curve $\alpha: I \longrightarrow \mathrm{IR}^{m}$ is said to be regular if $\alpha^{\prime}(t) \neq 0$ for all $t \in I$. Otherwise, the curve is said to be singular, and then, if $\alpha^{\prime}(\bar{t})=0$ for $\bar{t} \in I$, the image for this parameter value, $\alpha(\bar{t})$, is said to be a singular point or singularity.

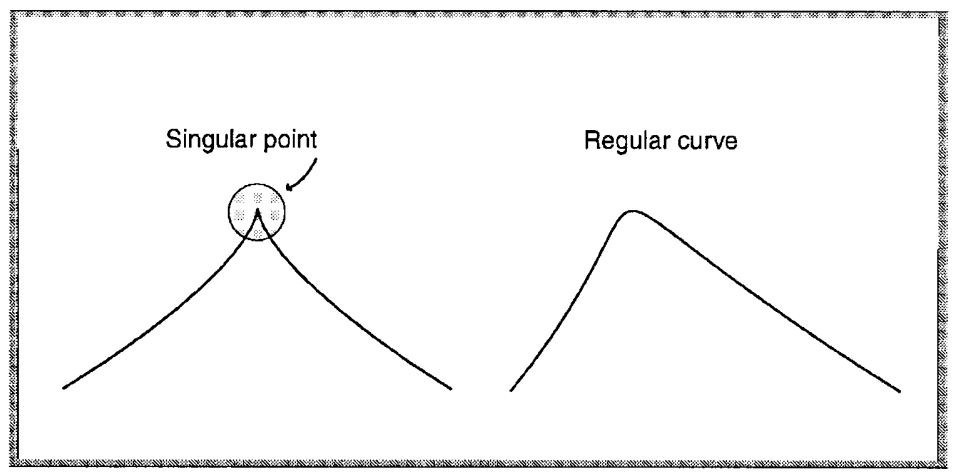

Figure 1 
Given the control points $\left\{D_{i}\right\}_{i=0}^{n}$ and weights $\left\{w_{i}\right\}_{i=0}^{n}$, let $X(t)$ be the open and clamped Nurbs curve of order $k$ associated to a knot vector

$$
T=\left(t_{0}=t_{1}=\ldots=t_{k-1}<t_{k}<\ldots<t_{n}<t_{n+1}=t_{n+2}=\ldots=t_{n+k}\right) .
$$

In this section we will found that if a Nurbs curve has a singular point $X(\bar{t})$, at the parameter value $\bar{t} \in\left[t_{r}, t_{r+1}\right]$, then it belongs to both previous curves of order $k-1$ at the de Boor algorithm, i.c., $D_{r-1}^{k-2}(t)$ and $D_{r}^{k-2}(t)$.

Theorem 1. Let $X(t)$ be an open and clamped Nurbs curve of order $k$ with control points $\left\{D_{i}\right\}_{i=0}^{n}$, weights $\left\{w_{i}\right\}_{i=0}^{n}$ and associated to a knot vector $T$.

If $X^{\prime}(\bar{t})=0$ for $\bar{t} \in\left[t_{r}, t_{r+1}\right]$ where $r \in\{k-1, \ldots, n\}$, then

$$
X(\bar{t})=D_{r}^{k-1}(\bar{t})=D_{r}^{k-2}(\bar{t})=D_{r-1}^{k-2}(\bar{t}) .
$$

Proof. First of all, we apply the recursive definition of the normalized B-Spline functions $N_{i, k}$ to the definition (2) of a Nurbs curve, then, shifting the indices in the second term by $i=i-1$ and taking $D_{-1}=D_{n+1}=0$ we get

$$
\begin{gathered}
X(t)=\frac{\sum_{i=0}^{n} w_{i} D_{i} N_{i, k}(t)}{\sum_{i=0}^{n} w_{i} N_{i, k}(t)} \\
=\frac{t\left(\sum_{i=0}^{n+1} \frac{w_{i} D_{i}-w_{i-1} D_{i-1}}{t_{i+k-1}-t_{i}} N_{i, k-1}(t)\right)+\sum_{i=0}^{n+1} \frac{w_{i-1} D_{i-1} t_{i+k-1}-w_{i} D_{i} t_{i}}{t_{i+k-1}-t_{i}} N_{i, k-1}(t)}{t\left(\sum_{i=0}^{n+1} \frac{w_{i}-w_{i-1}}{t_{i+k-1}-t_{i}} N_{i, k-1}(t)\right)+\sum_{i=0}^{n+1} \frac{w_{i-1} t_{i+k-1}-w_{i} t_{i}}{t_{i+k-1}-t_{i}} N_{i, k-1}(t)} .
\end{gathered}
$$

Having in mind (7), the de Boor algorithm and property 1 of the normalized B-Spline functions, we evaluate (9) at $\bar{t} \in\left[t_{r}, t_{r+1}\right]$ and we obtain that:

$$
X(\bar{t})=\frac{\frac{\bar{t} A^{\prime}(\bar{t})}{k-1}+\sum_{i=1}^{n} w_{i}^{1}(0) D_{i}^{1}(0) N_{i, k-1}(\bar{t})}{\frac{\bar{t} w^{\prime}(\bar{t})}{k-1}+\sum_{i=1}^{n} w_{i}^{1}(0) N_{i, k-1}(\bar{t})} .
$$

Moreover, formula (6) let us assert that if $X(\bar{t})$ is a singular point at the parameter value $\bar{t} \in\left[t_{k-1}, t_{n+1}\right]$, then

$$
X(\bar{t})=\frac{A^{\prime}(\bar{t})}{w^{\prime}(\bar{t})},
$$

so we can establish the following relation

$$
\frac{\frac{\bar{t} A^{\prime}(\bar{t})}{k-1}+\sum_{i=1}^{n} w_{i}^{1}(0) D_{i}^{1}(0) N_{i, k-1}(\bar{t})}{\frac{\bar{t} w^{\prime}(\bar{t})}{k-1}+\sum_{i=1}^{n} w_{i}^{1}(0) N_{i, k-1}(\bar{t})}=\frac{A^{\prime}(\bar{t})}{w^{\prime}(\bar{t})}
$$

hence

$$
X(\bar{t})=\frac{\sum_{i=1}^{n} w_{i}^{1}(0) D_{i}^{1}(0) N_{i, k-1}(\bar{t})}{\sum_{i=1}^{n} w_{i}^{1}(0) N_{i, k-1}(\bar{t})}
$$


Now if we apply again the recurrence formula for the normalized B-Spline functions and we use the blossoming principle we get

$$
\begin{gathered}
X(\bar{t})=\frac{\sum_{i=1}^{n} w_{i}^{1}(0) D_{i}^{1}(0) N_{i, k-1}(\bar{t})}{\sum_{i=1}^{n} w_{i}^{1}(0) N_{i, k-1}(\bar{t})} \\
=\frac{\sum_{i=1}^{n} \frac{\left(t_{i+k-2}-\bar{t}\right) w_{i-1}^{1}(0) D_{i-1}^{1}(0)+\left(\bar{t}-t_{i}\right) w_{i}^{1}(0) D_{i}^{1}(0)}{t_{i+k-2}-t_{i}} N_{i, k-2}(\bar{t})}{\sum_{i=1}^{n} \frac{\left(t_{i+k-2}-\bar{t}\right) w_{i-1}^{1}(0)+\left(\bar{t}-t_{i}\right) w_{i}^{1}(0)}{t_{i+k-2}-t_{i}} N_{i, k-2}(\bar{t})} \\
=\frac{\sum_{i=1}^{n} w_{i}^{2}[0, \bar{t}] D_{i}^{2}[0, \bar{t}] N_{i, k-2}(\bar{t})}{\sum_{i=1}^{n} w_{i}^{2}[0, \bar{t}] N_{i, k-2}(\bar{t})} .
\end{gathered}
$$

Therefore, the re-indexing can be continued by repeated insertion of the recurrence formula for the basis functions, and also, we can apply the blossoming principle for the $k-1$ steps, so we obtain

$$
\begin{aligned}
X(\bar{t}) & =\frac{\sum_{i=1}^{n} w_{i}^{k-1}\left[0, \bar{t}, . .{ }^{(k-2)} . ., \bar{t}\right] D_{i}^{k-1}\left[0, \bar{t}, . .{ }^{(k-2)} . ., \bar{t}\right] N_{i, 1}(\bar{t})}{\sum_{i=1}^{n} w_{i}^{k-1}\left[0, \bar{t}, . .{ }^{(k-2)} . ., \bar{t}\right] N_{i, 1}(\bar{t})} \\
& =D_{r}^{k-1}\left[0, \bar{t}, . .{ }^{(k-2)} . ., \bar{t}\right] .
\end{aligned}
$$

Hence, the above result let us to establish the following relation

$$
X(\bar{t})=D_{r}^{k-1}\left[\bar{t}, . .{ }^{(k-1)} . ., \bar{t}\right]=D_{r}^{k-1}\left[0, \bar{t}, . .{ }^{(k-2)} . ., \bar{t}\right] .
$$

Taking into account the symmetry of the blossom functions

$$
D_{r}^{k-1}\left[0, \bar{t}, . .{ }^{(k-2)} . ., \bar{t}\right]=D_{r}^{k-1}\left[\bar{t}, . .{ }^{(k-2)} . ., \bar{t}, 0\right],
$$

and otherwise its recursive definition, then, from the equality

$$
D_{r}^{k-1}\left[\bar{t}, . .{ }^{(k-1)} \ldots, \bar{t}\right]=D_{r}^{k-1}\left[\bar{t}, . .{ }^{(k-2)} . ., \bar{t}, 0\right]
$$

we can obtain the following relation

$$
D_{r-1}^{k-2}\left[\bar{t}, . .{ }^{(k-2)} . ., \bar{t}\right]=D_{r}^{k-2}\left[\bar{t}, . .{ }^{(k-2)} . ., \bar{t}\right] .
$$

On the other hand, applying again the symmetry at (10), we get

$$
\begin{gathered}
X(\bar{t})=D_{r}^{k-1}\left[\bar{t}, .{ }^{(k-1)} . ., \bar{t}\right]=D_{r}^{k-1}\left[\bar{t}, . .{ }^{(k-2)} . ., \bar{t}, 0\right]= \\
=\frac{\left(t_{r+1} w_{r-1}^{k-2}\left[t, . .{ }^{(k-2)} . ., t\right]-t_{r} w_{r}^{k-2}\left[t, .{ }^{(k-2)} . ., t\right]\right) D_{r}^{k-2}\left[t, .{ }^{(k-2)} . ., t\right]}{t_{r+1} w_{r-1}^{k-2}\left[t, .{ }^{(k-2)} . ., t\right]-t_{r} w_{r}^{k-2}\left[t, . .{ }^{(k-2)} . ., t\right]} \\
=D_{r}^{k-2}\left[\bar{t}, . .{ }^{(k-2)} . ., \bar{t}\right] .
\end{gathered}
$$


Therefore

$$
X(\bar{t})=D_{r}^{k-1}\left[\bar{t}, . .{ }^{(k-1)} . ., \bar{t}\right]=D_{r}^{k-2}\left[\bar{t}, .{ }^{(k-2)} . ., \bar{t}\right]=D_{r-1}^{k-2}\left[\bar{t}, . .{ }^{(k-2)} \ldots, \bar{t}\right],
$$

that is

$$
X(\bar{t})=D_{r}^{k-1}(\bar{t})=D_{r}^{k-2}(\bar{t})=D_{r-1}^{k-2}(\bar{t})
$$

Figure 2 illustrates an open B-Spline curve $X(t)$ of order 4 with control points $\left\{D_{i}\right\}_{i=0}^{5}$ and uniform knot vector $T=(0,0,0,0,1,2,3,3,3,3)$ which has a singular point for the parameter value $\bar{t} \in\left[t_{4}, t_{5}\right]=[1,2]$.

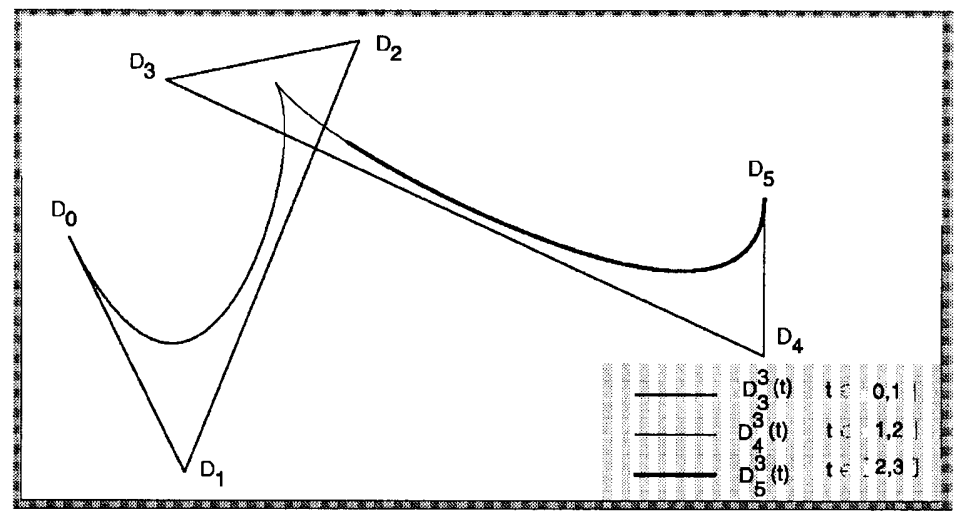

Figure 2

Figure 3 illustrates both previous curves to $X(t)=D_{4}^{3}(t)$ in the de Boor algorithm. These curves, $D_{4}^{2}(t)$ and $D_{3}^{2}(t)$, are represented in this picture only for parameter values on the interval $\left[t_{4}, t_{5}\right]=[1,2]$, and we can observe that they are tangent at the singular point $X(\bar{t})$.

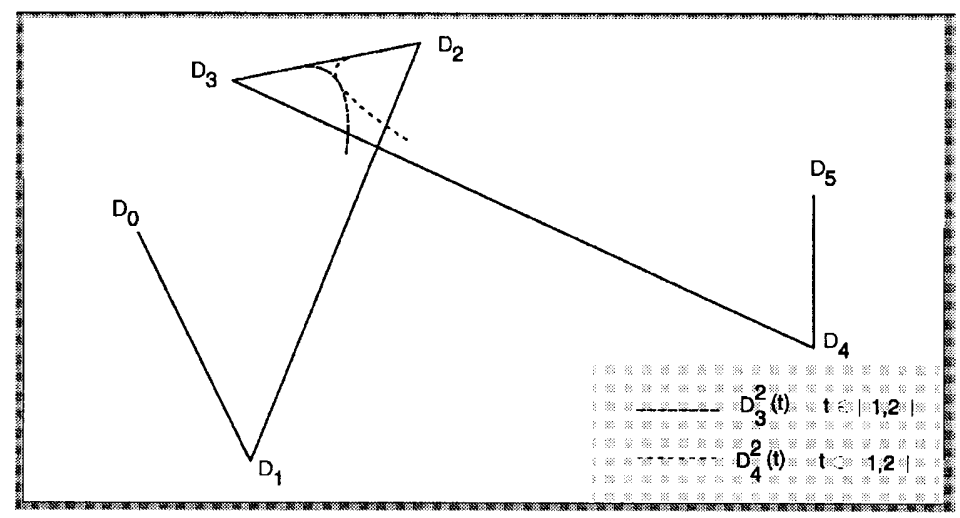

Figure 3 
Figure 4 illustrates the three curves described above, $X(t)=D_{4}^{3}(t)$, the B-Spline curve and also, $D_{4}^{2}(t)$ and $D_{3}^{2}(t)$ for the parameter values at $\left[t_{4}, t_{5}\right]=$ $[1,2]$.

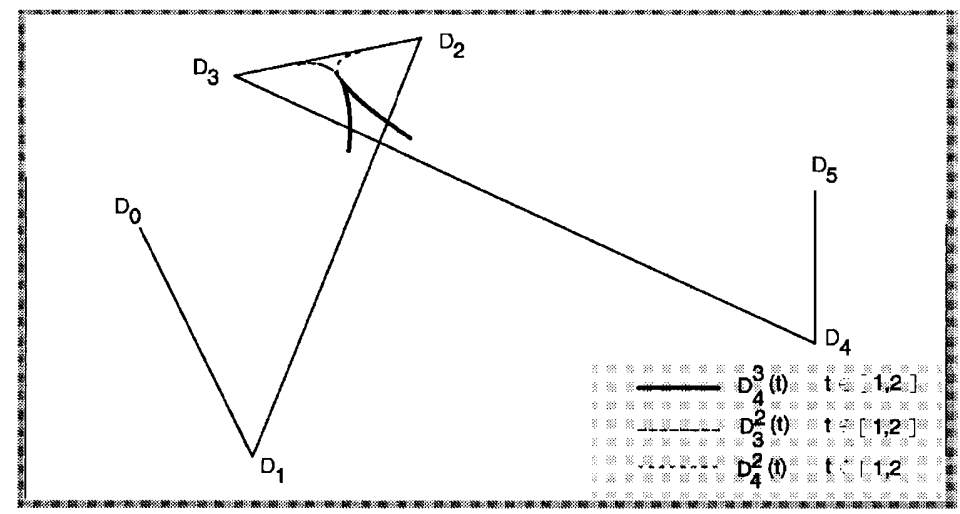

Figure 4

The following theorem proves that, also in the Nurbs curves case, both previous curves to a singular curve $X=D_{r}^{k-1}$ in the de Boor algorithm are tangent at this point.

Theorem 2. Let $X(t)$ be an open and clamped Nurbs curve of order $k$ with control points $\left\{D_{i}\right\}_{i=0}^{n}$, weights $\left\{w_{i}\right\}_{i=0}^{n}$ and associated to a knot vector $T$.

Let us suppose that $X(t)$ is singular at the parameter value $\bar{t} \in\left[t_{r}, t_{r+1}\right]$, then if the curves $\beta_{1}=D_{r}^{k-2}$ and $\beta_{2}=D_{r-1}^{k-2}$ are regular at $\bar{t}$, they are tangent at that point.

Proof. The last step of the de Boor algorithm for constructing Nurbs curves involves

$$
X(t)=\frac{\left(1-\alpha_{r}^{k-1}(t)\right) w_{r-1}^{k-2}(t) D_{r-1}^{k-2}(t)+\alpha_{r}^{k-1}(t) w_{r}^{k-2}(t) D_{r}^{k-2}(t)}{\left(1-\alpha_{r}^{k-1}(t)\right) w_{r-1}^{k-2}(t)+\alpha_{r}^{k-1}(t) w_{r}^{k-2}(t)}
$$

where

$$
\alpha_{r}^{k-1}(t)=\frac{t-t_{r}}{t_{r+1}-t_{r}}
$$

An straightforward computation at $t=\bar{t}$ of $X^{\prime}$, applying Theorem 1 (that is $D_{r}^{k-2}(\bar{t})=D_{r-1}^{k-2}(\bar{t})$ ), we get

$$
a(\bar{t})\left(D_{r}^{k-2}\right)^{\prime}(\bar{t})+b(\bar{t})\left(D_{r-1}^{k-2}\right)^{\prime}(\bar{t})=0
$$

where

$$
\begin{aligned}
a(t)= & \left(1-\alpha_{r}^{k-1}(t)\right)^{2} w_{r-1}^{k-2}(t)^{2} \\
& +\left(1-\alpha_{r}^{k-1}(t)\right) \alpha_{r}^{k-1}(t) w_{r-1}^{k-2}(t) w_{r}^{k-2}(t) \\
b(t)= & \left(1-\alpha_{r}^{k-1}(t)\right) \alpha_{r}^{k-1}(t) w_{r-1}^{k-2}(t) w_{r}^{k-2}(t)+\alpha_{r}^{k-1}(t)^{2}
\end{aligned}
$$

that is $\beta_{1}^{\prime}(\bar{t})$ and $\beta_{2}^{\prime}(\bar{t})$ are proportional, so $\beta_{1}$ and $\beta_{2}$ are tangent at $X(\bar{t})$. 
Taking into account that Nurbs curves are a generalization of B-Spline curves choosing weights $w_{i}=1 \forall i$, we can assert the following corollaries for B-Spline curves and so for Bézier curves.

Corollary 1. Let $X(t)$ be an open and clamped B-Spline curve of order $k$ with control points $\left\{D_{i}\right\}_{i=0}^{n}$ and associated to a knot vector $T$.

If $X^{\prime}(\bar{t})=0$ for $\bar{t} \in\left[t_{r}, t_{r+1}\right]$ where $r \in\{k-1, \ldots, n\}$, then

$$
X(\bar{t})=D_{r}^{k-1}(\bar{t})=D_{r}^{k-2}(\bar{t})=D_{r-1}^{k-2}(\bar{t}) .
$$

i.e, the singular point belongs to both previous curves to $X(t)$ at the de Boor algorithm, and moreover if both curves are regular at $t=\bar{t}$, then they are also tangent at that point.

Remark 2. Along this note we have considered a non uniform and clamped knot vector to get an open Nurbs curve, nevertheless, we could also consider open curves with unclamped knot vectors. In fact, all the results obtained above can be casily gencralized for non uniform and unclamped Nurbs curves.

The following corollary, proved in [5], particularizes the Nurbs case theorem to Bézier curves.

Corollary 2. Let us suppose that the Bézier curve $\alpha=B\left[P_{0}, \ldots, P_{n}\right]$ is not regular and let $\alpha(\bar{t})$ be a singular point, then

$$
\alpha(\bar{t})=B\left[P_{0}, \ldots, P_{n-1}\right](\bar{t})=B\left[P_{1}, \ldots, P_{n}\right](\bar{t}),
$$

2.e., the singular point belongs to both previous curves to $B\left[P_{0}, \ldots, P_{n}\right]$ in the de Casteljau algorithm, and moreover if they are regular at the parameter value $\bar{t}$, then they are tangent at that point.

\section{Conclusions}

The main results on this note let us assert that if an open Nurbs curve of order $k$ has a singular point, then it belongs, analogously to the rational Bézier case, to both curves of order $k-1$ defined in the $k-2$ step of the de Boor algorithm, and also that these curves are tangent at the singular point. These results are generalizations of some of the properties obtained in [5] for rational Bézier curves.

Differently from previous works which aim is to detect singularities, this note characterizes them in terms of the control points involved in the de Boor algorithm. This new approach results in the possibility of constructing curves with such singular points.

Then, one of the applications of these results, would be the possible construction of a singular shape using just one Nurbs curve (or B-Spline curve), instead of obtaining the desired singularity by joining regular Nurbs curves with the singular point at the juncture, and this would lead us to a computational saving. The following figure shows how using one Bézier curve, instead of two regular Bézier curves to obtain a singular shape, would let to reduce the number of points required to define the curve. 


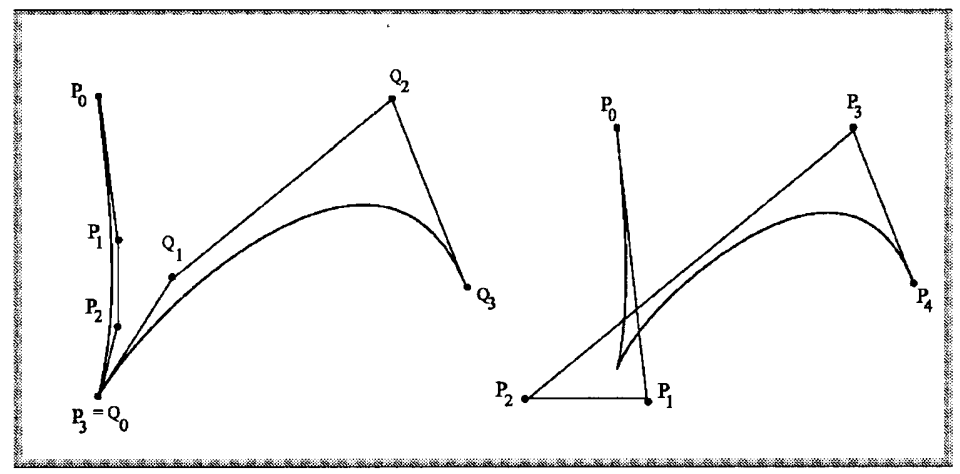

Figure 5

\section{Acknowledgements}

We would thank the referees for their valuable comments and suggestions.

\section{References}

1. S. Bu-Qing and L. Ding-Yuan, Computational Geometry. Curve and Surface Modeling, Academic Press, Inc., San Diego, 1989.

2. D. Manocha and J. F. Canny, Detecting cusps and inflection points in curves, Computer Aided Geometric Design 9, 1-24 (1992).

3. G. Farin, Curves and surfaces for computer aided geometric design. A practical guide, Morgan Kaufmann, 5th. ed. (2001).

4. J. Hoscheck and D. Lasser, Fundomentals of Computer Aided Geometric Design, A.K.Peters, Wellesley,1993.

5. J. Monterde, (2001), Singularities of rational Bézier curves, Computer Aided Geometric Design, 18, 805-816.

6. L. Piegl and W. Tiller, The Nurbs Book, 2a Ed., Springer Verlag, Berlin, 1997.

7. M. Sakai, (1999), Inflection Points and Singularities on Planar Rational Cubic Curve Segments, Computer Aided Geometric Design, 16, 149-156. 